\title{
A retrospective study of prognostic factors in locally advanced cancer of uterine cervix in a Rural Medical College of West Bengal
}

\author{
Dr. Santanu Acharya ${ }^{1}$, Dr. Ashis Kumar Saha ${ }^{2}$, Dr Bidyut Mandal ${ }^{1}$, \\ Dr Sumitava De ${ }^{1}$, Dr Srikrishna Mandal ${ }^{1}$, Dr. Chhanda Das ${ }^{3}$ \\ ${ }^{I}$ (Department of Radiotherapy, B.S. Medical College, Bankura, India) \\ ${ }^{2}$ (Department of Surgery, B.S. Medical College, Bankura, India) \\ ${ }^{3}$ (Department of Pathology, R.G. Kar Medical College, Kolkata, India.)
}

\begin{abstract}
Cervical cancer is the most common gynaecological malignancy in rural Bengal. There is significant improvement in pelvic disease control and survival when concurrent Cisplatin chemotherapy is added to radiotherapy in stage IB2 -IVA. We assessed the result of cervical cancer treated for the consecutive two years on total 189 patients, with particular emphasis on the methods of treatment and their prognostic outcome. The patients were evaluated with physical and pelvic examination for staging of cervical cancer, routine blood count, Biochemistry profile, chest radiograph, IVU, barium enema and CECT scan. Patients were treated with EBR to pelvis delivered with AP/ PA portal over five weeks followed by three HDR intracavitary brachytherapy for three consecutive weeks. Weekly concurrent Cisplatin chemotherapy was administered to seventy one patients. Patients were followed up every three months for the first two years, every four months for the third year, every six months for the fourth and fifth year and yearly afterwards. The observed 2 year survival was $66.90 \%$. Clinical stage at presentation was the single most important variable in predicting survival. The 2 year observed survival for stage II disease was $76.19 \%$, for stage III $54.76 \%$ and for stage IV $5.7 \%$.
\end{abstract}

Keywords: External Beam Radiotherapy, Intra Venous Urography, Contrast Enhanced Computed Tomography scan.

\section{Introduction}

Cervical cancer is the most common gynaecological malignancy in rural Bengal. The standard protocol for treatment is intracavitary brachytherapy combined with external beam radiotherapy. There is significant improvement in pelvic disease control and survival when concurrent Cisplatin chemotherapy is added to radiotherapy in stage IB2 -IVA cervical cancer ${ }^{[1-4]}$. Although concurrent chemo-radiotherapy followed by intracavitary brachytherapy is standard treatment paradigm for locally advanced cervical cancer. Most of our patients were treated with concurrent chemo-radiotherapy but few patients were treated with radiotherapy alone for compromised renal function or other co morbidity or patient's refusal.

\section{Aims And Objectives}

In this study, we assessed the result of cervical cancer treated in our department for the consecutive two years with particular emphasis on the methods of treatment and their prognostic outcome.

\section{Materials And Methods}

Total 189 patients were treated for the carcinoma cervix, of varied stages of IB2 to IVA in the department of radiotherapy, with External Beam Radiotherapy (EBRT) followed by intra-cavitary brachytherapy, between September 2008 and August 2010. Forty four patients were lost to follow up and three patients were died due to other co morbidity. The remaining 142 patients were included for study. The patients were previously untreated and had histological diagnosis of squamous cell carcinoma or adenocarcinoma.

Exclusion criteria:

1. Post hysterectomy patients.

2. Patients treated out side or lost to follow up.

3. Previously treated for cancer of the cervix

4. Karnofsky Performance Score less than 50

5. Renal dysfunction (Serum creatinine $<2.0 \mathrm{mg} / \mathrm{dl}$ )

6. Hepatic dysfunction (Serum bilirubin $<1.2 .0 \mathrm{mg} / \mathrm{dl}$, Transaminases $<1.5$ times normal). 
The median age of patients was 57 year (range 25-70 years). Seventy one patients were treated with concurrent chemotherapy. The patients were evaluated with physical examination and pelvic examination for staging of cervical cancer, routine blood count, blood Biochemistry profile, chest radiograph, Intra Venous Urography (IVU) and barium enema. Contrast Enhanced Computed Tomography (CECT) scan was used only for detecting pelvic and para -aortic lymphadenopathy. Pelvi and para- aortic lymph node more than $10 \mathrm{~mm}$ in minimum diameter considered to be metastatic lymphnode by CT scan. Neither lymphangiography nor the surgical evaluation of lymphnode was performed.

External beam radiotherapy: Patients were treated with external beam radiation to whole pelvis using ${ }^{60} \mathrm{Co} \mathrm{Y}$ rays with AP-PA portal. The median EBRT dose was to whole pelvis was $49 \mathrm{~Gy}$ in conventional fractionation. The irradiated volume was to include the uterus, para-cervical and para-metrium and uterosacral region as well as the hypogastric, obturator lymphnodes, external, internal and common iliac group of lymph nodes. The upper and lower border of EBRT portal passes though the L4/L5 junction of vertebra and lower border of obturator foramen respectively. The lateral border of AP/PA external beam radiotherapy portal passes through $2 \mathrm{~cm}$ beyond the bony pelvis.

Ten patients, who had para-aortic lymphnode involved in CECT scan, received para-aortic nodal irradiation in addition to whole pelvis radiotherapy. Seventy one patients were treated with concurrent platinum based chemotherapy.

Intracavitary brachytherapy: Usually three intra cavitary insertion using HDR $\operatorname{Ir}_{192}$ isotope (Varian Medical system, Gammamed) was performed in every week after completion of external beam radiotherapy. Brachytherapy was delivered by After Loading Technique and Manchester Applicators has been used. Applicator insertion usually done under intravenous anaesthesia and anterior and posterior vaginal packing was done using radio opaque gauge piece to displace the bladder wall and rectum from high dose region. Patients were treated in dorsal lithotomy position. External fixation device was used to prevent applicator movement. Median dose delivered to Point A, $700 \mathrm{cGy}$ in each fraction of brachytherapy and total three insertions for each patient. Point A was defined on radiograph as being $2 \mathrm{~cm}$ superior (along the uterine tandem) to the flange abutting the external cervical Os and $2 \mathrm{~cm}$ lateral from the axis of the tandem. The rectal and bladder reference points were defined on radiograph according to the ICRU report 38.

The treatment schedule: External beam radiotherapy to pelvis delivered with AP/ PA portal to a total dose of 50 Gy in 25 fractions in five weeks followed by three HDR intracavitary brachytherapy of 7 Gy in weekly insertion for three consecutive weeks. Weekly platinum based concurrent chemotherapy was administered to seventy one patients (cisplatin $40 \mathrm{mg} / \mathrm{M}^{2}$ ).

Follow up: Both radiation oncologist and gynaecologist revive the patients every three months for the first two years, every four months for the third year, every six months for the fourth and fifth year and yearly afterwards. Those patients who were lost to follow up were excluded from the statistical analysis. Follow up procedure includes general and systemic examination and routine pelvic examination. Loco regional recurrence was suspected by pelvic examination and or papanicolaou smear and biopsy was taken for confirmation. Radiological examination of abdomen or chest ware performed as clinically indicated. Late urinary bladder and rectal complication were graded accordance with NCI- CTC ver 2.

Statistical analysis: Overall survival of the entire group was calculated from the interval between the first date of EBRT and last date of follow up or patient's death and was plotted using Kaplan Meier method. Difference in incidence of toxicity were analysed by chi square test and survival by log rank test.

\section{Results And Analysis}

Between September 2008 and August 2010, a total of one hundred eighty nine patients were treated for the carcinoma cervix of stage IB2 to IVA in the department of radiotherapy with external beam radiotherapy followed by intracavitary brachytherapy. Results were analyzed in April 2012. Three cervical cancer patients were died due to other co morbid condition and forty four patients were lost to follow up. After a median follow up period of twenty three months (range 5 months to 39 months), forty four out of one hundred forty two patients eventually had disease progression and died after palliative chemotherapy. About seventy six percent (32 out of 42) of stage II B and fifty four percent (46 out of 84) of stage III cervical cancer survived in a median follow up period twenty three months (range 5 months to 39 months). Nine patients with cervical cancer had para-aortic lymphadenopathy and only one patient was survived even after thirty four months. Twenty five (83.3\%) patients with cervical cancer with pelvic lymphadenopathy had developed loco regional/distant recurrence after nine to sixteen months post treatment. Three patients died due distant failure only. Thirty three percent of patients had developed loco-regional/distant failure those who had no pelvic lymphadenopathy in median follow up period twenty three months. Twenty one patients died due to loco-regional failure and remaining patients died due to loco regional and distant or isolated distant failure. Fifty nine percent patients in concurrent chemo-radiotherapy arm survived where as fifty three percentages patients in radiotherapy arm remained alive in a median follow up period of twenty three months. Age is also important prognostic factor in cervical cancer. Fifty four percent patients survived in group with age less than fifty four years whereas fifty eight percent patients for those who were older than fifty four years. Late rectal (radiation proctitis) and urinary bladder (radiation cystitis) toxicity were the most common adverse events noted. Only seven patients $(5 \%)$ had developed Late Grade 3 radiation induced proctitis. Grade 2 and Grade 1 radiation induced proctitis was noted 
in $6.9 \%(10 / 142)$ and $58 \%(82 / 142)$ respectively. Late radiation induced bladder toxicity was noted in $27.30 \%$ (39/142) of cases and 3.5\% (5/142) patients had experienced Grade 3 adverse events. Diarrhea was the most common GI toxicity and was experienced by 14 out all 142 patients (9.5\%), and was easily managed with anti diarrheal therapy.

\section{Figures And Tables}

Table-1: Profile of the patients affected by Cancer of uterine cervix $(\mathrm{N}=142)$

\begin{tabular}{|c|c|c|c|c|c|}
\hline \multicolumn{3}{|c|}{ Characteristics } & \multirow{2}{*}{$\begin{array}{c}\text { Number } \\
70\end{array}$} & \multirow{2}{*}{$\begin{array}{c}\text { Percentage } \\
49.29\end{array}$} & \multirow{2}{*}{$\begin{array}{l}\text { Statistics }\left(\chi^{2} \text { of }\right. \\
\text { Goodness of fit, df, } p) \\
0.02,1,>0.05\end{array}$} \\
\hline \multirow{2}{*}{\multicolumn{2}{|c|}{ Age (yr) }} & $<56$ & & & \\
\hline & & $\geq 56$ & 72 & 50.70 & \\
\hline \multirow{2}{*}{\multicolumn{2}{|c|}{ Tumour size $(\mathrm{cm})$}} & $<40$ & 69 & 48.59 & \multirow[t]{2}{*}{$0.08,1,>0.05$} \\
\hline & & $\geq 40$ & 73 & 51.41 & \\
\hline \multirow{3}{*}{\multicolumn{2}{|c|}{ Stage of disease }} & IIB & 42 & 29.58 & \multirow[t]{3}{*}{$35.02,2,<0.05$} \\
\hline & & III & 84 & 59.15 & \\
\hline & & IVA & 16 & 11.27 & \\
\hline \multirow{4}{*}{$\begin{array}{l}\text { Lymph } \\
\text { node } \\
\text { involvement }\end{array}$} & \multirow[t]{2}{*}{ Pelvic } & Present & 30 & 21.13 & \multirow[t]{2}{*}{ NA } \\
\hline & & Absent & 112 & 78.87 & \\
\hline & \multirow[t]{2}{*}{ Paraaortic } & Present & 9 & 6.34 & \multirow[t]{2}{*}{ NA } \\
\hline & & Absent & 133 & 93.66 & \\
\hline \multirow{2}{*}{\multicolumn{2}{|c|}{ Treatment }} & Radiotherapy & 71 & 50.00 & \multirow[t]{2}{*}{ NA } \\
\hline & & $\begin{array}{l}\text { Radiotherapy + } \\
\text { Chemotherapy }\end{array}$ & 71 & 50.00 & \\
\hline
\end{tabular}

$\mathrm{df}=$ degree of freedom, $* \mathrm{NA}=$ not applied

Table-2: Distribution of overall survival of patients as per their profile $(\mathrm{N}=142)$.

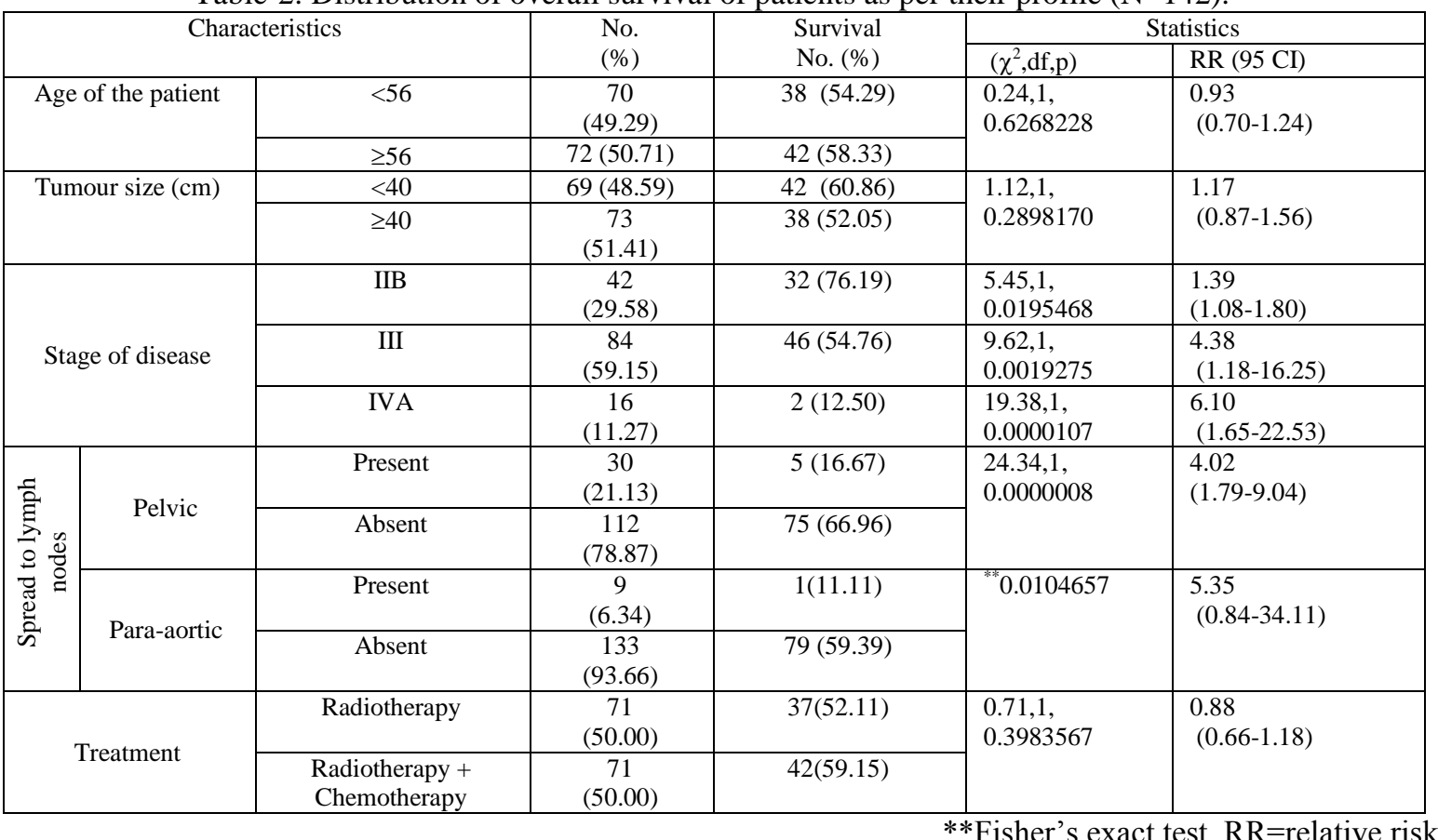

\section{Discussion}

Cancer of the cervix is the most common cancer among women in India, constituting between onesixth to one-half of all female cancers with an age-adjusted incidence rate ranging from 19.4 to 43.5 per 100,000 in the registries under the National Cancer Registry Programme (NCRP) (Annual Reports, NCRP, ICMR $)^{[5]}$. It has been estimated that 100,000 new cases of cancer of the cervix occur in India every year, and $70 \%$ or more of these are Stage III or higher at diagnosis. Besides incidence and clinical stage at presentation knowledge of survival is essential to complete the picture of establishing baseline indicators to monitor and evaluate outcome of cervical cancer. However, because locally advanced cervical cancer usually accompanied by high risk factors such as surrounding tissue infiltration, lympho vascular embolism, regional lymph node metastasis, the efficacy of radiotherapy alone has not improve for years. The reasons might relate to the tumor pathological type, size, more hypoxic cell, radiation dose and limitation of normal tissue tolerance. As a result, current treatment modality lays emphasis on the improvement of treatment intensity and combined treatment 
which consist of radiotherapy with concurrent chemotherapy or biological therapy ${ }^{[6]}$. During the last 10-15 years, the GOG, RTOG and SWOG group have published plenty of randomized controlled trial and had showed that concurrent chemotherapy improve response rate and reduced mortality of loco regional advanced cervical cancer. The observed 2 year survival was $66.90 \%$. Clinical stage at presentation was the single most important variable in predicting survival. The 2 year observed survival for stage II disease was $76.19 \%$, for stage III $54.76 \%$ and for stage IV $5.7 \%$.

Our result showed that compared to radiotherapy group over all survival of chemo-radiotherapy group was higher but statistically not significant $(\mathrm{P}=0.398)$. Stage based stratified analysis showed that the difference in the over all survival between the radiotherapy and chemo radiotherapy arms were statically significant. In developing countries like India, where chemo radiation can be afforded by a minority only, judicious use of radiotherapy still produces satisfactory results with acceptable toxicity. The addition of chemotherapy may be beneficial in patients with adverse prognostic factors. Age is an important prognostic factor in cervical cancer but failed to reach statistically significance. Age at diagnosis emerged as important prognostic factor in cervical cancer but failed to reach statistically significant ${ }^{[7]}$.

The results obtained in our study are also in agreement with multiple clinical observations on pelvic and para aortic nodal metastasis in cervical cancer ${ }^{[8]}$. The patients with pelvic and para-aortic lymphadenopathy had poorer prognosis and higher loco regional or metastatic rates and had statically significant poorer survival (P-0.00000008, P-0.0104) (Table 2).

Although the addition of concurrent chemotherapy to radiation undoubtedly has benefited many patients, there is still considerable room for improvement. Some patients, particularly those with bulky tumors or regionally advanced disease, continue to experience local recurrences, suggesting the need for even more effective chemo radiotherapy regimens. However, investigators face major challenges in trying to design future chemo radiotherapy trials as because: 1) The fact that Cisplatin was included in the most successful arms of so many trials has caused investigators to question whether it is ethical to omit cisplatin or even to compromise the cisplatin dose in new chemo radiotherapy regimens ${ }^{[9]}$.2) The hematologic toxicity of weekly cisplatin and of the combination of Cisplatin and 5FU limits the dose that can be safely delivered in combination with radiation. For this reason, one attractive approach for future studies will be the incorporation of relatively non-myelosuppressive biologic response modifiers into current regimens. Recent interest has focused on epidermal growth factor modulators and vascular endothelial growth factor modulators, cyclooxygenase-2 inhibitors, and agents that specifically target hypoxic cells ${ }^{[10]}$. Despite the challenges, we must also continue to evaluate potentially radiosensitizing drugs that could prove to be more effective than cisplatin or equally effective but less toxic or less costly. In doing so, we should consider concurrent chemo radiotherapy regimens that have proved to be successful against tumors at other sites-particularly carcinomas of the head and neck, which have response characteristics that are in many ways similar to those of cervical cancers. Conversely, although preradiation chemotherapy has been used with some success in other sites, randomized trials have repeatedly failed to show a benefit from neoadjuvant chemotherapy in patients with cervical cancer. Although some investigators have suggested sequencing neoadjuvant chemotherapy and chemo radiotherapy, the possibility that preradiation induction chemotherapy could compromise the intensity of subsequent chemo radiotherapy, diminishes the attractiveness of this approach.

\section{REFERENCES}

[1] Whitney CW, Sause W, Bundy BN, Malfetano JH. Randomized comparison of fluorouracil plus cisplatin versus hydroxyurea as an adjunct to radiation therapy in stage IIB-IVA carcinoma of the cervix with negative para-aortic lymph nodes: a Gynecologic Oncology Group and Southwest Oncology Group study. J Clin Oncol. 1999 May;17(5):1339-48

[2] Eifel PJ, Winter K, Morris M. Pelvic irradiation with concurrent chemotherapy versus pelvic and para-aortic irradiation for highrisk cervical cancer: an update of radiation therapy oncology group trial (RTOG) 90-01. J Clin Oncol. 2004 Mar 1;22(5):872-80

[3] Rose PG, Ali S, Watkins E, Thigpen JT, Deppe G; Long-term follow-up of a randomized trial comparing concurrent single agent cisplatin, cisplatin-based combination chemotherapy, or hydroxyurea during pelvic irradiation for locally advanced cervical cancer: a Gynecologic Oncology Group Study. J Clin Oncol. 2007 Jul 1;25(19):2804-10

[4] Keys HM, Bundy BN, Stehman FB, Okagaki T, Gallup DG; Radiation therapy with and without extrafascial hysterectomy for bulky stage IB cervical carcinoma: a randomized trial of the Gynecologic Oncology Group. Gynecol Oncol. 2003 Jun;89(3):343-53

[5] Nandakumar A, Anantha N, Venugopal TC. Incidence, mortality and survival in cancer of the cervix in Bangalore, India. . Br J Cancer. 1995 Jun;71(6):1348-52

[6] Wang S, Zhang DS, Pan T, Liu S, Wang MK. Efficacy of concurrent chemoradiotherapy plus adjuvant chemotherapy on advanced cervical cancer. Chin J Cancer. 2010 Nov;29(11):959-63

[7] Gajalakshmi V, Rajaraman S, Shanta V. A survival study of cervical cancer in Chennai, India. Indian J Cancer. 2000 Dec;37(4):158-64

[8] Stehman F, Bundy B, DiSaia PJ, et al. Carcinoma of the cervix treated with irradiation therapy: A multi-variate analysis of prognostic variables in the Gynecologic Oncology Group. Cancer 67:2776-2785

[9] Thomas GM: Improved treatment for cervical cancer--concurrent chemotherapy and radiotherapy. N Engl J Med 340 (15): 1198 200, 1999

[10] Ishikawa H, Ohno T, Kato S, Wakatsuki M. Cyclooxygenase-2 impairs treatment effects of radiotherapy for cervical cancer by inhibition of radiation-induced apoptosis. Int J Radiat Oncol Biol Phys. 2006 Dec 1;66:1347-55 